\title{
STUDIES ON BIOLUMINESCENCE.
}

XVII. Fluorescence and Inhibition of Luminescence in CtenoPHORES BY UltRA-VIOLET Light.

BY E. NEWTON HARVEY.

(From the Physiological Laboratory, Princeton University, Princeton, the Nela Research Laboratories, Cleveland, and the Marine Biological

Laboratory, Woods Hole.)

(Accepted for publication, October 21, 1924.)

It is possible to distinguish three types of luminous animals, in regard to their behavior toward illumination. Some forms (Chrtopterus, Ptylosarcus (1)) when stimulated, are capable of luminescence on removal to a dark room immediately after exposure to daylight, while others (Mnemiopsis, Bolina, Renilla (2)) do not luminesce under these circumstances. Their power of luminescence is inhibited by daylight but is regained when kept in the dark for a short time. Still other forms (Pelagia (3)) show a persistent day and night rhythm of luminescence. Their luminescence is inhibited by light but if kept continually in a dark room they luminesce on stimulation only during the period corresponding to night and not during the period corresponding to day.

The behavior toward light of ctenophores, which belong in the second group, has been known for a long time. The inhibition of luminescence by sunlight was first described by Allman and has been further studied by Panceri (5), Peters (6), Harvey (1), and Moore (7). The sudden appearance of the Ctenophore, Mnemiopsis leidyi, at Woods Hole during the latter part of August and September gave an opportunity to test certain questions concerning the inhibition of luminescence by light.

As is well known, the luminous areas in Mnemiopsis leidyi are restricted to the eight rows of paddle plates (meridians) andmechanical stimulation of these anywhere will cause a luminescence at the point touched. Often a wave of luminescence will pass along the 
meridian from the region stimulated. Stimulation of regions between the paddle plates (intermeridians) does not cause luminescence unless deformation of the tissue affects the paddle plate region (Moore (7)). The general nerve net work is not connected with the luminous cells and the only fact indicating that conducting pathways are involved is the propagation of the luminescence along the meridian. The propagation does not cross a cut dividing the meridian into two halves.'

Isolated bits of meridian tissue will give light on stimulation, but Peters (6) was never able to obtain light from a piece with less than four paddle plates. I have seen luminescence from stimulation of the tissue attached to two paddle plates. The important point is to have some of the canal present, for the luminous cells are located within the canal (Dahlgren (8)).

Small bits of canal tissue will not necessarily give light on mechanical agitation although they contain luminescent material, as can be shown by treatment with tap water or saponin, when luminescence will occur. This was demonstrated in conjunction with Professor Robert Chambers, who, with his micro dissection apparatus, dissected out a clump of canal cells and placed a glass dissecting needle in the midst of them. When this needle was moved back and forth no light appeared, but when some saponin solution was injected among them with a micro pipette, numerous luminous points appeared, similar to those observable in luminescent colenterates in general (1). Pieces of canal tissue of considerable length will give light if a micro needle is moved about in the tissue. It looks as if mechanical agitation causes luminescence readily only when a considerable mass of tissue, probably containing some nerve connections, is present.

Pieces of canal tissue with a few paddle plates attached will lose their power of luminescence in light and regain it in the dark, and the question arises, how small may fragments of ctenophores be and still show the characteristic behavior towards illumination?

\footnotetext{
${ }^{1}$ In some individuals propagation of a wave of light does not take place readily, but in others it does. There seems to be no doubt, however, but that a luminescence wave is really propagated along the canal, and that we are not merely dealing with a mechanical deformation of the canal tissue along its entire length.
} 
If a dark adapted ctenophore is shaken in sea water and the sea water filtered through filter paper, an opalescent solution is obtained, which gives a few isolated points of light on shaking. When fresh water is added to it or a little saponin powder, many bright points of light appear, a phenomenon that may for convenience be called, "starry luminescence." The solution is opalescent and the centrifuge separates many single cells, often ciliated, and globular cell fragments, clearly seen under the microscope. The fresh water or saponin probably causes cytolysis of cells ${ }^{2}$ containing luminous material.

This filtered extract of dark adapted Mnemiopsis behaves toward light as the whole animal. Its power of luminescence on adding tap water is lost after illumination and returns again in the dark, although the recovery in the dark is not complete. The amount of starry luminescence is never so great after exposure to sunlight as before.

Conversely, the extract ${ }^{3}$ of a light adapted ctenophore, after standing in sunlight for 10 minutes, gives no luminescence when immediately brought into a dark room and tap water added, but upon standing in the dark for 10 minutes, the addition of tap water calls forth the starry luminescence.

We are therefore led to the conclusion that isolated photogenic cells (and perhaps fragments) that will pass through filter paper, have luminescent material actually decomposed by sunlight and reformed in the dark. The experiments confirm the conclusion I arrived at with Bolina $a^{4}$ (1), that sunlight acts on the photogenic

${ }^{2}$ Or perhaps a dissolving of photogenic granules, granulolysis.

${ }^{3}$ Made by squeezing a ctenophore through cheese-cloth. Often bits of tissue with four or five paddle plates come through the cheese-cloth. After standing in the dark these luminesce readily on slight agitation but the great mass of finely divided fragments do not luminesce on agitation, although they readily luminesce on adding fresh water.

${ }^{4}$ A light adapted Mnemiopsis squeezed through cheese-cloth in the dark always gives some faint luminescence but never as great a luminescence as is obtained on treating a dark adapted animal in the same way. The light adapted Bolina extract will not recover its luminescent power in the dark (1) but the Mnemiopsis extract will. Mnemiopsis contains much more photogenic material than Bolina and this may explain the difference. 
material itself, and supplement those of Moore (7) who finds that filter paper drawn over the meridians will take up material that luminesces when vigorously rubbed, or placed in isotonic $\mathrm{KCl}$ solution. This luminescent paper loses its power of luminescence in sunlight or artificial light but a much longer exposure is required than in the case of the whole animal. Moore interprets this to mean that in the animal light acts to inhibit luminescence through a nerve mechanism.

The nerve connections are strictly local, for I can confirm Moore's observation that only the region of a Mnemiopsis exposed to light is inhibited. The non-illuminated areas luminesce readily on stimulation and the line of demarcation between the two regions is very sharp if the beam of light used for illumination is well defined.

Light certainly acts directly on the photogenic material, even if it has in addition a more effective action through a nerve mechanism, and the question arises whether light inhibits luminescence by oxidizing the photogenic substance. One might suppose that in daylight a ctenophore luminesced and used up its available supply of material. We could not see this luminescence in the glare of day. Inhibition would thus be comparable to fatigue which occurs so readily upon repeated stimulation of the animal. ${ }^{5}$ The question could be tested by observation of the ctenophore in a phosphoroscope, but as a phosphoroscope was not available, I have made use of ultra-violet (without the visible) light.

The spectral region which inhibits luminescence is not known with any certainty but one might predict the violet end of the spectrum to be most efficient. ${ }^{\circ}$ In order to determine if the near ultra-violet has any power of inhibiting luminescence, a number of experiments

${ }^{5}$ It should be noted that a ctenophore shaken in a bottle for 10 minutes until only slight flashes of light appear, still has much photogenic material in it, as considerable luminescence occurs when squeezed through cheese-cloth, and the extract gives a brilliant starry luminescence in tap water. The fatigue in question is probably a nerve fatigue, not a complete exhaustion of photogenic material.

- A few experiments indicate that the violet end of the spectrum is much more efficacious than the red end in inhibiting luminescence. Time did not permit an accurate study of the relation. 
were carried out with a quartz mercury lamp, ${ }^{7}$ the Hanovia 110 volt A. C. Alpine Sunburner, using 720 to 740 watts after running this for some time. The animals were exposed at a distance of from 4.5 to 5.5 inches in glass dishes behind various filters which allow the near ultra-violet to pass. The far ultra-violet was of course cut out by the glass dish and filters.

If a dark adapted Mnemiopsis, which is brightly luminescent on stimulation, is placed in the light from the quartz mercury lamp filtered through a No. 18 Wratten ultra-violet filter, the luminescence, on stimulation at intervals, will be found to diminish gradually and in 30 minutes will have practically disappeared, even on violent stimulation. An animal in the dark, stimulated at the same intervals, will be found to be markedly luminescent at each stimulation. Hence the inhibition of luminescence is not due to fatigue but to the action of the ultra-violet light, similar in its action to sunlight. Practically no visible light passes a No. 18 filter, at least not enough to inhibit luminescence of Mnemiopsis, as a much greater light from a tungsten lamp in the room will not inhibit the luminescence.

Although sunlight is more effective in inhibiting the luminescence of Mnemiopsis, the beauty of experiments in ultra-violet light is that one can see whether the animal will luminesce during exposure, since the animal's luminescence on stimulation is perfectly apparent and very bright as compared with the slight amount of visible light which passes the No. 18 filter, or the small amount of fluorescent light from near objects. The method is therefore beautifully adapted to answer the question previously raised, whether light stimulates the animals to luminesce and uses up the available photogenic material.

Observation of the Mnemiopsis before the ultra-violet lamp with No. 18 filter shows that no luminescence appears, provided the animal is not disturbed. The animal is dark except for a slight bluish fluorescence along the swimming plates. If kept undisturbed in this ultra-violet light, no signs of luminescence appear over a

7 I take great pleasure in thanking Dr. S. E. Pond for the use of the mercury lamp and for sharing the Nela Laboratory dark room in which many of these experiments were carried out. 
period of 30 minutes, when the animal has lost completely its power of luminescence on stimulation.

Ultra-violet light does not stimulate to luminescence but a very interesting phenomenon appears when the animal is mechanically stimulated in the ultra-violet. If stimulated several times a very decided bluish luminescence persists after the luminescence due to stimulation has subsided. This "tonic luminescence," as it may be called, disappears when the ultra-violet is screened and reappears when the ultra-violet again strikes the animal. It behaves like a fluorescence. The animal may be stimulated in the dark and when placed in the ultra-violet the tonic luminescence is observed. It lasts for some time while the animal is in the ultra-violet light, but gradually fades out.

There are two possible explanations of the tonic luminescence. (1) It is a true chemiluminescence due to continuous or tonic stimulation of the photogenic cells in the ultra-violet light, after their luminescence has been started by mechanical stimulation. (2) The product of oxidation of photogenic material is fluorescent.

It is difficult to decide between these views. Certainly the behavior of this tonic luminescence is like a fluorescence, appearing only in the exciting radiation, but at the same time it is always associated with potentially luminescent animals. Light adapted animals (i.e. those exposed to sunlight) do not show tonic luminescence when stimulated before the ultra-violet. Therefore, the effect is not due to fluorescence of any non-luminescent mucus, secreted along the paddle plates on stimulation. Observation with a microscope shows that the tonic luminescence is in the canal, in the same position as the true luminescence of the animal. It seems always to be associated with once luminous material.

Animals partially fatigued by gentle shaking for 2 minutes in a bottle show tonic luminescence in the ultra-violet. Ctenophores so treated are fatigued for all practical purposes, since they give only an occasional faint flash of light. If shaken in a bottle for 15 minutes, when luminescence on stimulation is no longer possible, the tonic luminescence in ultra-violet is no longer apparent. It appears as if some material must have diffused out into the sea water when agitated for a long time. Indeed sea water in which luminescent 
ctenophores have been shaken is markedly fluorescent, much more so than ordinary sea water, but fluorescence of organic material is so general that one cannot necessarily conclude that in this case we are dealing entirely with the fluorescence of photogenic substances.

On the whole, I believe the evidence points to the second explanation as correct, that the tonic luminescence is a fluorescence of the oxidation product or some intermediate substance, formed during luminescence of the animal. This view is borne out by the fact that Chatopterus, a luminous worm, produces a luminous slime which is very markedly fluorescent in near ultra-violet light. Moreover, the Chatopterus slime collected on a glass rod quite apart from the animal, is notably fluorescent, so that we are not dealing with any stimulation mechanism that may be set off in ultra-violet light. On the other hand Cypridina luciferin, oxyluciferin, and luciferase are not markedly fluorescent in the near ultra-violet, although I have often endeavored to observe some sign of fluorescence similar to that obtained with Chatopterus.

The luminous organs of the glowworm show marked fluorescence which is of a different color (more yellowish) from that of the fatbody, and of the luciferesceine, found throughout the body of the insect (see Dubois (9) and McDermott (10)). It will be of considerable interest to test other luminous animals for strong fluorescence of photogenic material.

More and more evidence is accumulating to indicate a close relation between fluorescence and chemiluminescence. Kautsky and Zocher (11) have pointed out the relations in unsaturated silicon compounds, and have shown (12) that fluorescent dyes adsorbed on non-chemiluminescent compounds (such as silico-oxalic acid) become luminescent on treatment with oxidizing agents. Evans and Dufford (13), and Dufford, Calvert, and Nightingale (14) find that many of the Grignard compounds (organomagnesium halides) are notably chemiluminescent, and their oxidation products are fluorescent in ultra-violet light. Esculin is fluorescent and most of the essential oils that are chemiluminescent in alcoholic caustic alkali, are also fluorescent.

Perhaps it is not surprising, then, to find fluorescence accompanying the brilliant photogenic material of luminous animals. 
SUMMARY.

1. Small clumps of the luminous cells of Mnemiopsis cannot readily be stimulated mechanically but will luminesce on treatment with saponin solution. Larger groups of luminous cells (such as are connected with two paddle plates) luminesce on mechanical stimulation. This suggests that mechanical stimulation to luminesce occurs chiefly through a nerve mechanism which has been broken up in the small clumps of luminous tissue.

2. The smallest bits of luminous tissue, even cells freed from the animal by agitation, that will pass through filter paper, lose their power to luminesce in daylight and regain it (at least partially) in the dark.

3. Luminescence of the whole animal and of individual cells is suppressed by near ultra-violet light (without visible light).

4. Inhibition in ultra-violet light is not due to stimulation (by the ultra-violet light) of the animal to luminesce, thereby using up the store of photogenic material.

5. Animals stimulated mechanically several times and placed in ultra-violet light show a luminescence along the meridians in the same positions as the luminescence that appears on stimulation. This luminescence in the ultra-violet or "tonic luminescence," is not obtained with light adapted ctenophores and is interpreted to be a fluorescence of the product of oxidation of the photogenic material.

6. Marked fluorescence of the luminous organ of the glowworm (Photuris) and of the luminous slime of Chatopterus may be observed in ultra-violet but no marked fluorescence of the luminous substances of Cypridina is apparent.

7. Evidence is accumulating to show a close relation between fluorescent and chemiluminescent substances in animals, similar to that described for unsaturated silicon compounds and the Grignard reagents.

\section{BIBLIOGRAPHY.}

1. Harvey, E. N., Biol. Bull., 1921, xli, 280.

2. Parker, G. H., Proc. Am. Phil. Soc., 1920, xix, 171.

3. Heymans, C., and Moore, A. R., J. Gen. Physiol,, 1923-24, vi, 273.

4. Allman, G. 1., Proc. Roy. Soc. Edinburgh, 1862, iv, 518. 
5. Panceri, P., Atti. Roy. Accad. Sc. Napoli, 1872, v, No. 20.

6. Peters, A. W., J. Exp. Zool., 1905, ii, 103.

7. Moore, A. R., J. Gen. Physiol., 1923-24, vi, 403.

8. Dahlgren, U., J. Franklin Inst., 1916, clxxxi, 243.

9. Dubois, R., Bull. Soc. Zool. France, 1886, xi, 1.

10. McDermott, F. A., J. Am. Chem. Soc., 1911, xxxiii, 410.

11. Kautsky, H., and Zocher, H., Z. Physik, 1922, ix, 267.

12. Zocher, H., and Kautsky, H., Naturwissenschaften, 1923, xi, 194.

13. Evans, W. V., and Dufford, R. T., J. Am. Chem. Soc., 1923, xlv, 278.

14. Dufford, R. T., Calvert, S., and Nightingale, D., J. Am. Chem. Soc., 1923, $\mathrm{lxv}, 2058$. 